\title{
Nonthermal solid-to-solid phase transitions in tungsten
}

\author{
Yvelin Giret, ${ }^{1,2}$ Szymon L. Daraszewicz, ${ }^{1}$ Dorothy M. Duffy, ${ }^{1}$ Alexander L. Shluger, ${ }^{1}$ and Katsumi Tanimura ${ }^{2}$ \\ ${ }^{1}$ Department of Physics and Astronomy, University College London, Gower Street, WC1E 6BT London, United Kingdom \\ ${ }^{2}$ Institute of Scientific and Industrial Research (ISIR), Osaka University, Mihogaoka 8-1, Ibaraki, Osaka 567-0047, Japan
}

(Received 3 June 2014; revised manuscript received 2 August 2014; published 4 September 2014)

\begin{abstract}
The ab initio calculations of phonon dispersions and nonthermal forces along structural deformation paths were used to study nonthermal solid-to-solid phase transitions in photoexcited tungsten. We assumed that electronic excitation can be described by an electronic temperature and demonstrated that nonthermal, i.e., caused purely by electronic excitation, bcc-to-fcc and bcc-to-hcp phase transitions can occur for electronic temperatures between 1.7 and $4.3 \mathrm{eV}$. These transitions result from soft modes along the $\Sigma$ line of the Brillouin zone. Structural path calculations at different electronic temperatures indicate that both transitions are likely to take place in nonequilibrium conditions. We further predict that transient fcc and hep phases of tungsten could be observed for several ps.
\end{abstract}

DOI: 10.1103/PhysRevB.90.094103

PACS number(s): $61.50 . \mathrm{Ks}, 71.15 . \mathrm{Qe}, 71.15 . \mathrm{Mb}, 71.15 . \mathrm{Nc}$

\section{INTRODUCTION}

Electronic excitation of a solid by femtosecond laser pulses can create a transient nonequilibrium state in which the electron subsystem is "hot" and the ion subsystem remains close to its initial temperature $[1,2]$. Such excitation modifies the electron density distribution in a solid giving rise to modified forces between atoms. The modified interatomic forces, in turn, can cause coherent atomic motions and structural transitions on a very short (subpicosecond) time scale [3-5]. Such phase transitions occur without invoking significant energy transfer between electrons and ions and are therefore referred to as nonthermal phase transitions. Concurrently, the energy absorbed by the electronic subsystem dissipates into the ionic degrees of freedom via electron-phonon coupling, causing the ionic temperature to increase. The ionic temperature can rise beyond the melting point, which would result in the disordering of the lattice in few ps. This transition is called ultrafast thermal melting. In contrast, the so-called nonthermal melting caused by change in the character of electron density distribution (chemical bonds) occurs more rapidly and without significant increase of the ionic temperature. The desire to understand and control such rapid phase transitions has given rise to extensive research including studies on molecular switching [6-9], nonthermal melting [10-13], coherent phonon generation [14-17], metal-to-insulator [18-20], magneticto-nonmagnetic [21-23], and other ultrafast solid-to-solid [24-26] phase transitions. In this paper we focus on solid-tosolid phase transitions in simple metals, particularly tungsten.

Experimentally, ultrafast phase transitions are studied with time-resolved techniques, such as time-resolved optical spectroscopy, x-ray and electron diffraction, and two-photon photoemission, which allow one to track different transient properties of the irradiated material. Recently, ultrafast solidto-solid phase transitions have been demonstrated in several materials: the metal-to-insulator transitions in a number of vanadium oxides [18,20,27] and in manganites [25,28], and the neutral-to-ionic transitions in organic TTF-CA crystals [6,7,9,29]. However, time-resolved studies of phase transitions are challenging as they require simultaneous access to femtosecond dynamics of several degrees of freedom of the system. Here we propose a simple theoretical approach to assess the possibility of inducing a nonthermal solid-to-solid phase transition by a femtosecond laser pulse. The method is applicable to wide range of metals.

To theoretically describe nonthermal phase transitions, and more generally ultrafast phase transitions, it is necessary to invoke some model of the electronic excitation. Following absorption of the laser pulse energy, an electronic subsystem does not have a well-defined energy distribution, but rather tends towards a Fermi-Dirac distribution (characterized by an electronic temperature $T_{e}$ ) through electron-electron collisions. We note here that a phase transition is referred to as nonthermal regardless of the details of the electronic distribution. A relatively rapid electron thermalization in metals [30] (10-100 fs) can lead to a cold plasma state (also referred to as warm dense matter) in which the electronic temperature is much greater than the ionic temperature (i.e., $T_{e} \gg T_{i}$ ) for longer than several ps. The lifetime of such a state is primarily determined by the effective electron-phonon coupling, and can vary by several orders of magnitude, depending on the material's characteristics, shape and size (bulk solid, thin film), temperature, and pressure [31-33].

The energy transfer between the electron and ion subsystems is often studied using a phenomenological twotemperature model (2TM) [30,34]. This model can describe the temporal evolution of the electronic and ionic temperatures and hence the lifetime of such a two-temperature state, but it cannot describe the associated atomic dynamics. A recently developed two-temperature molecular dynamics (2T-MD) model [35] couples the continuum description of the electronic subsystem in 2TM to classical dynamics of nuclei. This method has been successfully applied to describe the ultrafast thermal and nonthermal melting of metals after laser excitation $[31,36]$, but has not yet been used to study nonthermal solid-to-solid phase transitions. Indeed, describing nonthermal solid-to-solid phase transition in the $2 \mathrm{~T}-\mathrm{MD}$ model requires an interatomic potential that takes into account the existence of particular soft phonon modes in electronically excited metals. To the best of our knowledge, no such potential exists and its construction would require the determination of the different structural paths that become available to a particular material as a result of electronic excitation.

In this paper we demonstrate that one can use $a b$ initio calculations to assess the possibility of inducing nonthermal 
bcc-to-fcc and bcc-to-hcp phase transitions in tungsten by laser pulse. However, our modeling approach should be applicable to other metals where the description of electronic excitations in terms of electronic temperature is valid. The method can reliably predict the possibility of nonthermal structural transitions in metals screened for ultrafast light-switching functionality and is the first step towards a more complete physical description of the atomic dynamics involved in such transitions.

\section{CALCULATION DETAILS}

The $a b$ initio calculations are performed using the ABINIT code [37,38] in the local density approximation (LDA) [39] using the norm-conserving pseudopotential method [40], where the $5 d$ and $6 s$ electrons are retained as valence electrons. The valence pseudo-wave functions are expanded into plane waves with kinetic energy cutoff of $60 \mathrm{Ha}$. A $16 \times 16 \times 16$ Monkhorst-Pack $\boldsymbol{k}$-points mesh is used for the bcc and fcc structures, and a $12 \times 12 \times 6$ mesh is used for the hcp structure. In this framework, basic structural and electronic properties for the ground-state bcc structure are in good agreement with literature values. In particular, we obtain a cell parameter of $a_{0}=3.196 \AA$ compared to an experimental value of $3.16 \AA$ at room temperature. Dynamical matrices are computed on a $8 \times 8 \times 8 \boldsymbol{q}$-points grid in the Brillouin zone for bcc and fcc structures, and on a $6 \times 6 \times 3$ grid for the hep structure, and used for interpolation to obtain phonon spectra for different $T_{e}$ along high-symmetry directions.

Calculations at high electronic temperature implement the generalization of the density functional theory (DFT) [41,42] given by Mermin [43] to describe an electronic subsystem at thermal equilibrium at the electronic temperature $T_{e}$. In this formalism, the temperature of the electronic subsystem appears on the one hand through an entropic contribution $S_{e}$ to the free energy $A=E-T_{e} S_{e}$, and on the other hand through the Fermi-Dirac occupation function for the Kohn-Sham eigenstates $\varepsilon_{i}$ :

$$
f_{i}=\frac{1}{e^{\left(\varepsilon_{i}-\mu_{e}\right) / k_{B} T_{e}}+1},
$$

where $\mu_{e}$ is the chemical potential of the electrons and where $k_{B}$ is the Boltzmann's constant. Considering independent particles in the Kohn-Sham representation, the electronic entropy is written [44]

$$
S_{e}=-k_{B} \sum_{i=1}^{+\infty}\left[f_{i} \ln f_{i}+\left(1-f_{i}\right) \ln \left(1-f_{i}\right)\right],
$$

where the sum runs over occupied and unoccupied states.

In this context, the Kohn-Sham functional is replaced by the Mermin functional $[44,45]$ :

$$
\begin{aligned}
A\left[n,\left\{f_{i}\right\} ; T_{e}\right]= & T_{0}^{\beta}[n]+E_{H}^{\beta}[n]+\int d \boldsymbol{r} V_{\mathrm{ext}}(\boldsymbol{r}) n^{\beta}(\boldsymbol{r})+E_{x c}^{\beta}[n] \\
& -T_{e} S_{e}\left[\left\{f_{i}\right\}\right],
\end{aligned}
$$

where $E_{H}$ is the Hartree energy, $E_{x c}$ the exchange-correlation energy, and $V_{\text {ext }}$ the external potential acting on the electrons. The $\beta$ symbol indicates that the quantity is calculated at finite temperature. Below, we study the modification of interatomic forces at a given electronic temperature. One can show that, at a given $T_{e}$, the force $\boldsymbol{F}_{I}$ acting on the atom $I$ at a position $\boldsymbol{R}_{I}$ is given by the derivative of the Mermin functional [46-48]:

$$
\boldsymbol{F}_{I}\left(T_{e}\right)=-\frac{\partial A\left(T_{e}\right)}{\partial \boldsymbol{R}_{I}},
$$

which we call a nonthermal force.

\section{PHONON SPECTRUM CALCULATIONS}

Most elemental metals have a crystal structure that is either body-centered cubic (bcc) (which is also the case for tungsten) or a more close-packed structure, such as face-centered cubic (fcc) or hexagonal closed-packed (hcp) [49]. We focus on these three structures in our study of nonthermal structural phase transitions in tungsten. To assess whether a particular phase is dynamically stable under electronic excitation, we calculate phonon dispersion curves as a function of electronic temperature. This corresponds to a scenario where the electronic subsystem has absorbed the laser pulse energy and has thermalized to a Fermi-Dirac distribution without transferring energy to the ionic subsystem. A dynamical instability, also called mechanical instability in the literature [49], will manifest itself by imaginary phonon frequencies in some points of the Brillouin zone (BZ). For instance, in silicon a softening of the whole transverse acoustic branch at an elevated electronic temperature is considered as the origin of a nonthermal melting transition [50]. Similarly, a softening of the longitudinal optical branch at the $\Gamma$ point in bismuth is considered as the origin of coherent optical phonon generation [51,52]. A softening of a branch in the whole BZ will give rise to disordering of the lattice. However, a good candidate for a nonthermal solid-to-solid phase transition should exhibit a phonon softening along a well-defined direction in the BZ. In the case of tungsten, the $a b$ initio calculations of phonon dispersion curves as a function of pressure predicted the existence of soft modes along the $\Gamma-\mathrm{N}$ and $\mathrm{P}-\mathrm{H}$ lines together with the fcc structure becoming dynamically stable [53,54], and these soft modes were suggested as an explanation for the pressure-induced bcc-to-fcc transition. To assess the possibility of nonthermal solid-to-solid phase transitions, we calculate phonon dispersion curves in bcc, fcc, and hcp tungsten as a function of the electronic temperature.

Figure 1(a) shows the calculated phonon branches for bcc $\mathrm{W}$ at $T_{e}=300 \mathrm{~K}$ compared with the experimental results [55], where we obtain an excellent agreement in the whole Brillouin zone. Figure 1(b) shows the calculated phonon branches for bcc $\mathrm{W}$ at $T_{e}=300 \mathrm{~K}$ and at $T_{e}=20000 \mathrm{~K}$ where one can see the appearance of soft transverse modes $\left(T_{1}\right)$ along the $\Gamma$-N line (called $\Sigma$ line), with the eigenvectors $(1, \overline{1}, 0)$. We note that a similar softening of the $T_{1}$ mode along the $\Sigma$ branch with increasing pressure has been reported in Ref. [54], but this is much less pronounced than the softening of the longitudinal mode at $\boldsymbol{q}=\left[\frac{2}{3}, \frac{2}{3}, \frac{2}{3}\right]$ that connects the bcc phase to the $\omega$ phase. We also observe the softening of this mode as $T_{e}$ increases, but it is much less pronounced than the softening along the $\Sigma$ line. We note that different structural transformations have been proposed caused by a soft mode on the $\Gamma-\mathrm{N}$ line of the bcc Brillouin zone [54,56,57], particularly bcc-to-fcc and bcc-to-hcp transformations caused by the 

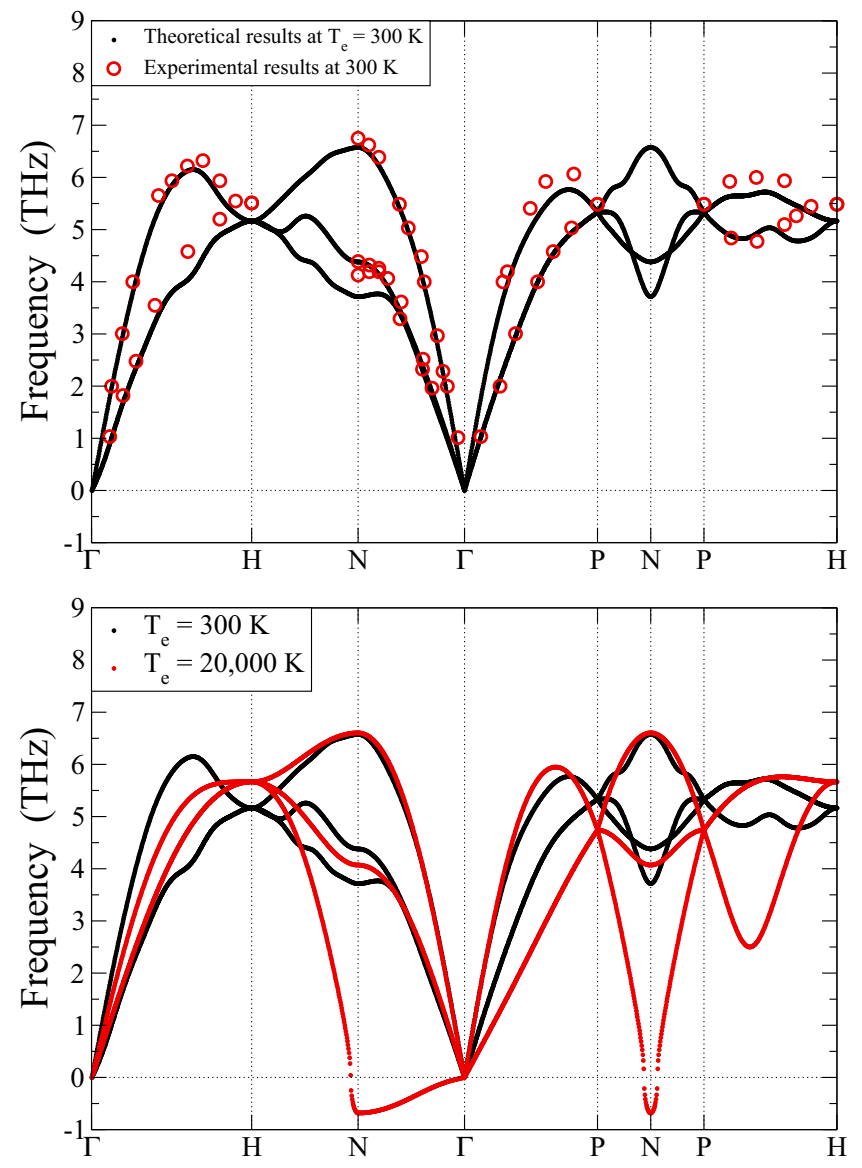

FIG. 1. (Color online) (a) Phonon spectrum of bcc W calculated for $T_{e}=300 \mathrm{~K}$ (black lines) compared with experimental results [55] (red circles). (b) Theoretical phonon spectra of bcc W for $T_{e}=300 \mathrm{~K}$ (black lines) and $T_{e}=20000 \mathrm{~K}$ (red lines) at the room temperature equilibrium volume $V_{0}^{e q}(300 \mathrm{~K})$.

soft modes $T_{[1, \overline{1}, 0]}\left[\frac{1}{4}, \frac{1}{4}, 0\right]$ and $T_{[1, \overline{1}, 0]}\left[\frac{1}{2}, \frac{1}{2}, 0\right]$. We would like to emphasize that these transformations are totally different from the nonthermal melting of silicon, where phonon softening is observed in the whole Brillouin zone [50].

Figure 2(a) shows the calculated phonon branches for fcc $\mathrm{W}$ at $T_{e}=300 \mathrm{~K}$ and $T_{e}=20000 \mathrm{~K}$ for a calculated lattice constant $a_{\mathrm{fcc}}^{\mathrm{eq}}(300 \mathrm{~K})=4.090 \AA$, corresponding to an increase of the equilibrium atomic volume of $\sim 4.8 \%$ compared to the bcc structure. These results show that fcc $\mathrm{W}$ is dynamically unstable at room temperature, in agreement with the literature $[53,54]$ and becomes dynamically stable as $T_{e}$ increases. Figure 2(b) shows the calculated phonon branches for hcp W at $T_{e}=300 \mathrm{~K}$ and at $T_{e}=20000 \mathrm{~K}$, for a calculated lattice constant $a_{\text {hcp }}^{\text {eq }}(300 \mathrm{~K})=2.866 \AA$ (with $c=\sqrt{8 / 3}$ assuming an "ideal" hexagonal close packed structure), corresponding to an increase of the equilibrium atomic volume of $\sim 2.0 \%$ compared to the bcc structure. As for the fcc structure, hcp $\mathrm{W}$ is dynamically unstable at $T_{e}=300 \mathrm{~K}$ and becomes progressively dynamically stable as $T_{e}$ increases, thus demonstrating similar behavior to the fcc structure. We note that both structures remain metallic, and that our results demonstrate that both fcc and hep tungsten
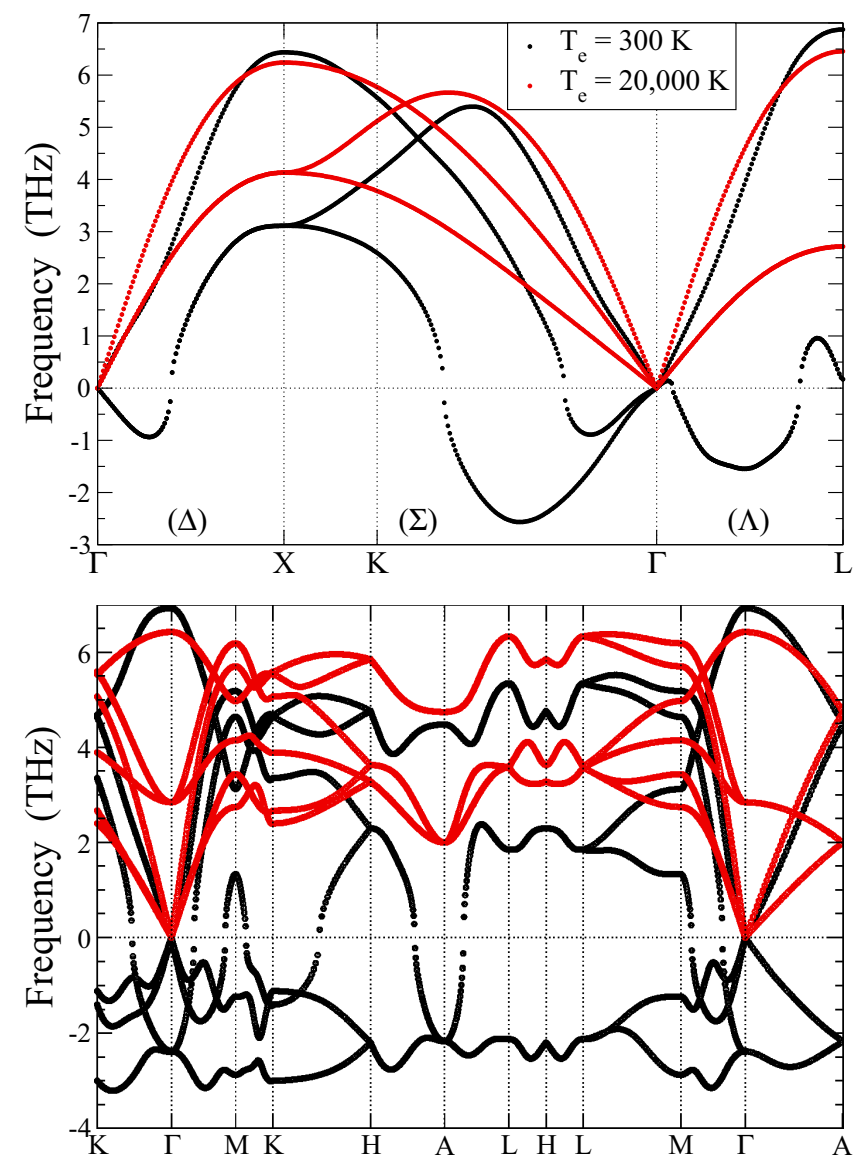

FIG. 2. (Color online) (a) Theoretical phonon spectra of fcc W for $T_{e}=300 \mathrm{~K}$ (black lines) and $T_{e}=20000 \mathrm{~K}$ (red lines) at room temperature equilibrium volume $V_{\mathrm{fcc}}^{e q}(300 \mathrm{~K})$. (b) Theoretical phonon spectra of hcp W for $T_{e}=300 \mathrm{~K}$ (black lines) and $T_{e}=20000 \mathrm{~K}$ (red lines) at the room temperature equilibrium volume $V_{\mathrm{hcp}}^{e q}(300 \mathrm{~K})$.

structures are stabilized by electronic entropy as dynamical stability is reached at high $T_{e}$.

We note that the equilibrium atomic volume $V_{\text {eq }}\left(T_{e}\right)$ increases when $T_{e}$ increases due to the electronic pressure applied to the lattice which may result in modified interatomic interactions. For instance, a hardening of all phonon branches at elevated electronic temperatures has been reported for gold, when calculated at the room-temperature atomic volume [50] $V_{\text {eq }}(300 \mathrm{~K})$, and a strong softening of all branches, when calculated [36] at $V_{\mathrm{eq}}\left(T_{e}\right)$. We performed calculations for both cases for $\mathrm{W}$ without noticing significantly different behavior. The calculations performed at $V_{\mathrm{eq}}(300 \mathrm{~K})$ assume that the lattice volume had no time to adjust to the electronic excitation, whereas those performed at $V_{\mathrm{eq}}\left(T_{e}\right)$ assume that the lattice had time to respond.

\section{STRUCTURAL TRANSFORMATION PATHS}

It is possible to further assess the dynamical stability of a particular phase and the possibility of a phase transition through the calculation of the energy change as a function of an external parameter, such as temperature or pressure, along structural deformation pathways. Examples of typical 
deformation pathways include the Bain paths connecting bcc and fcc structures and the Burgers paths connecting bcc and hcp structures [49]. In general, Bain paths are directly related to the elastic constants $C_{i j}$ of the solid, defined as the change of elastic energy associated with an arbitrary deformation given by small strains [49]. There are three independent elastic constants in a cubic solid, namely, $C_{11}, C_{12}$, and $C_{44}$. If we define the elastic shear constant by $C^{\prime}=\left(C_{11}-C_{12}\right) / 2$ and the bulk modulus by $B=\left(C_{11}+2 C_{12}\right) / 3$, it is possible to show [49] that a cubic lattice is dynamically stable if $B>0$, $C^{\prime}>0$, and $C_{44}>0$, criteria called Born stability criteria. The case $B<0$ is referred to as a spinodal instability [49] leading to a decohesion without lattice symmetry change. In our case, this will correspond to the electronic temperature at which the electronic free energy does not have a minimum. For tungsten, this electronic temperature is $T_{e}=50000 \mathrm{~K}(4.3 \mathrm{eV})$. This case does not interest us here as it cannot lead to a solid-to-solid phase transition. The case $C^{\prime}<0$ is referred to as the Born instability and is characterized by the symmetry breaking with coupling of shear modes under volume conservation, and $C_{44}<0$ is a pure shear instability [49].

When studying pressure-induced phase transitions, it is possible to calculate the total energy change along structural paths for different unit cell volumes. In general, the total energy does not include the incoherent ionic motion contribution as the phonon entropy is not well defined when an instability exists [53]. In this study we are interested in the change in instantaneous interatomic forces as a function of electronic temperature. As the forces acting on fixed atoms at a given electronic temperature are given by the change in the electronic free energy [see Eqs. (3) and (4)], we have calculated the change of total electronic free energy at different electronic temperatures along the structural deformation paths.

We first consider the tetragonal path, also called the Bain deformation path, which is the simplest continuous path between the bcc and the fcc lattice. The transformation corresponds to the stretching of the three sides in the cubic cell by factors of $x, x$, and $1 / x^{2}$, keeping the unit cell volume constant. The intermediate lattice has a tetragonal structure, with the crystallographic unit cell axes $a$ and $c$. Going from the bcc to the fcc lattice corresponds to varying $c / a$ from 1 to $\sqrt{2}$ in a body-centered tetragonal (bct) structure [49]. The elastic constant $C^{\prime}$ corresponds to the second derivative of the total energy at the point where $c / a=1$. Figure 3(a) shows the evolution of the electronic free energy along the tetragonal path for $T_{e}=300 \mathrm{~K}$ and $T_{e}=20000 \mathrm{~K}(1.7 \mathrm{eV})$. One can see that the fcc structure has lower energy than the bcc structure and that there is no energy barrier between the two structures. This case corresponds to a negative elastic shear constant $\left(C^{\prime}<0\right)$ where a crystalline solid would be dynamically unstable with respect to long-wavelength acoustic shear [53]. The negative curvature at $c / a=1$ will give rise to nonthermal forces that will tend to drive the originally bcc structure towards the fcc structure. These results show that photoexcited tungsten could undergo a nonthermal bcc-to-fcc phase transition if $T_{e}$ remains high enough for sufficient time.

We also calculated the trigonal Bain path that connects the bcc and the fcc lattices and that is directly related to the trigonal (or rhombohedral) shear modulus $C_{44}$. Starting with the bcc lattice, the trigonal path represents a homogeneous
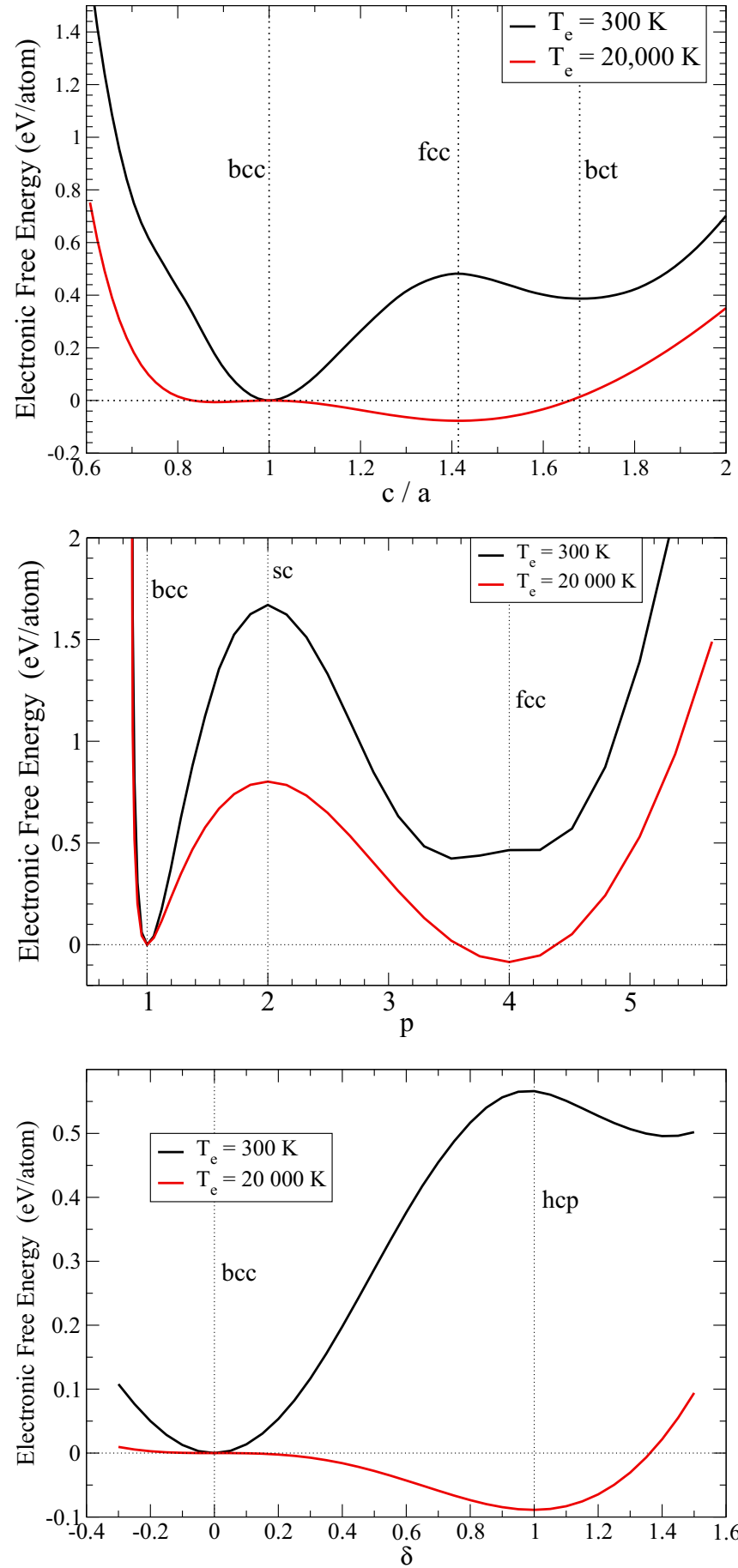

FIG. 3. (Color online) The electronic free energy change for (a) the tetragonal path connecting bcc and fcc structures for $T_{e}=$ $300 \mathrm{~K}$ (black lines) and $T_{e}=20000 \mathrm{~K}$ (red lines), (b) the trigonal path connecting bcc and fcc structures for $T_{e}=300 \mathrm{~K}$ (black lines) and $T_{e}=20000 \mathrm{~K}$ (red lines), and (c) the hexagonal path connecting bcc and fcc structures for $T_{e}=300 \mathrm{~K}$ (black lines) and $T_{e}=20000 \mathrm{~K}$ (red lines). (bcc stands for body-centered cubic, fcc for face-centerd cubic, bct for body-centered tetragonal, sc for simple cubic, and hcp for hexagonal close packed.)

deformation that corresponds to the extension along the [111] axis while keeping the unit cell volume fixed [58]. This path connects three cubic structures, and can be characterized by 
a parameter $p$, where $p=1$ corresponds to a bcc structure, $p=2$ to a simple cubic (sc) structure, $p=4$ to a fcc structure, and where $C_{44}$ is given by the second derivative of the total energy at the point $p=1$. Figure 3(b) shows the electronic free energy changes along the trigonal path between $T_{e}=300 \mathrm{~K}$ and $T_{e}=20000 \mathrm{~K}$. One can see that the fcc structure has lower energy than the bcc structure at high electronic temperature, but there is a potential barrier along this structural deformation path. These results suggest that photoexcited tungsten cannot undergo a nonthermal bcc-to-fcc phase transition through an electronically induced pure shear instability.

Finally, we have calculated a hexagonal path, characteristic of the Burgers transition mechanism, connecting bcc and hcp lattices. This path is a combination of a homogeneous deformation and a shuffling of alternate close-packed atomic planes in opposite directions. These two degrees of freedom may be coupled to obtain a one-parameter $(\delta)$ transformation path [59]. An intermediate structure along the path is an orthorhombic cell with four atoms. For $\delta=0$, we obtain the bcc structure and $\delta=1$ corresponds to the hcp structure with an ideal $(c / a)_{\text {hcp }}$ ratio of $\sqrt{8 / 3}$. Figure $3(\mathrm{c})$ shows the electronic free energy changes along the hexagonal path between $T_{e}=300 \mathrm{~K}$ and $T_{e}=20000 \mathrm{~K}$. We see a similar behavior to the electronic free energy changes along the Bain tetragonal path. These results show that high electronic temperature in tungsten gives rise to nonthermal forces that tend to simultaneously drive the bcc structure towards more closed-packed fcc and hcp structures.

In reality, the different physical processes involved are dynamical. Before laser excitation, the system is represented by $c / a=1, p=1$, and $\delta=0$ in Figs. 3(a), 3(b), and 3(c), respectively, and the energy landscape is represented by the black curves. After an instantaneous rise of the electronic temperature to $20000 \mathrm{~K}$, the system is described by the same parameters but the energy landscape shown by the red curves. As the atoms start to move driven by the nonthermal forces, the electronic temperature will decrease by the electron-phonon coupling and energy diffusion into the bulk. The energy landscape is then dynamically represented by free energy curves evolving between the two extremes given by $T_{e}=T_{e}^{\max }$ and $T_{e}=300 \mathrm{~K}$. The maximum electronic temperature will be given by the absorbed laser energy, and both the success of the nonthermal solid-to-solid phase transition and the time the system can spend in the new phase will be determined by the rate of excess energy redistribution.

\section{DISCUSSION}

Both the fcc and hep structures are dynamically stable below the electronic temperature at which the bcc phase becomes unstable. This corresponds to the electronic temperature range between $10000 \mathrm{~K}$ and $15000 \mathrm{~K}$, where we observe strong directional softening of the bcc lattice, but not yet an instability. We note here that the free energies of the fcc and hcp phases are already lower in this range of electronic temperature than the free energy of the bcc phase. Nonthermal forces will start to act on atoms only a few fs after the electron subsystem has absorbed the laser energy, and a nonthermal phase transition can occur in less than a phonon period [60]. Therefore, we can safely assume that the onset time for such transitions will be subpicosecond.

To estimate the lifetime of possible dynamical phases following laser irradiation, we have performed simulations based on the $a b$ initio-parametrized 2TM. Using the value of the effective electron-phonon coupling constant for tungsten $\left(G_{0}=1.65 \times 10^{17} \mathrm{~W} \mathrm{~m}^{-3} \mathrm{~K}^{-1}\right)$ and the calculated electronic heat capacity [61], we estimate that for a bulk sample, an absorbed fluence of $\sim 50 \mathrm{~mJ} \mathrm{~cm}{ }^{-2}$ could create the conditions where the electronic temperature would be high enough and the lattice temperature low enough in the first $\sim 50 \mathrm{~nm}$ from the irradiated surface for several ps (2-3 ps typically), giving sufficient time for both nonthermal phase transitions to occur before a possible ultrafast thermal melting. These estimates do not, however, take into account the fact that laser pulse excites only a small volume of the material and therefore a bcc to fcc/hcp phase transformation will be impeded by the interface with the rest of the solid. However, in the case of a thin film the electronic temperature will decrease more slowly than in the case of a bulk sample as the dissipation channel through electronic heat diffusion will not be available. The possibility of inducing a nonthermal, solid-to-solid phase transition for a given material will, therefore, depend on the conditions of a particular experiment.

To obtain some complementary information about the electronic behavior that causes lattice instabilities, we have calculated the electronic density difference $\Delta n(\boldsymbol{r})$ between $T_{e}=20000 \mathrm{~K}$ and $T_{e}=300 \mathrm{~K}$ (see Fig. 4). The increase of the electronic density in each of the Cartesian directions around all atoms suggests how the electronic density changes can give rise to lattice instabilities. However, a full study of the characteristics of the different electronic excited states goes beyond the scope of the present study.

It is known that pressure usually weakens directional forces and drives crystalline structures toward close-packed forms [62]. Our results suggest that the electronic temperature could have a similar general effect on $d$-band transition metals, as they have a strong directional component in their electronic bonding compared to other metals, which is affected by the excitation (see Fig. 4). We have performed similar calculations for tantalum, another approximately half-filled $d$-band transition bcc metal, and found that Ta exhibits the same kind of behavior as a function of $T_{e}$ as $\mathrm{W}$. On the other hand, previous calculations for $\mathrm{Au}$ have shown that Au undergoes phonon hardening as the electronic temperature increases [50], if the unit cell volume is kept at $V_{\mathrm{eq}}(300 \mathrm{~K})$,

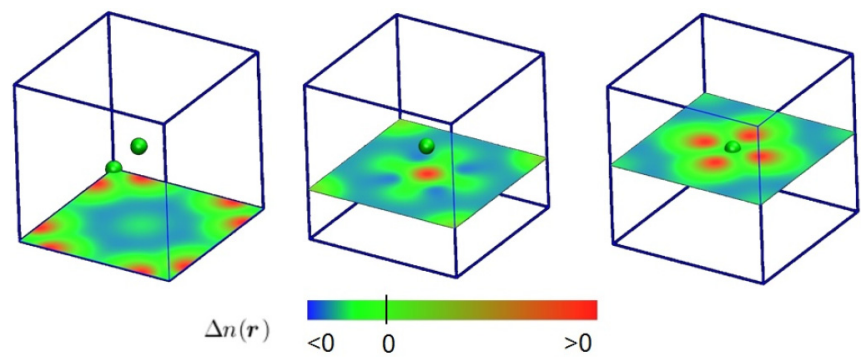

FIG. 4. (Color online) Electron density difference $\Delta n(\boldsymbol{r})$ between $T_{e}=20000 \mathrm{~K}$ and $T_{e}=300 \mathrm{~K}$ in the conventional cubic bcc unit cell. 
and phonon softening, if the unit cell volume is relaxed [36] at $V_{\text {eq }}\left(T_{e}\right)$. We have observed the same kind of behavior for other $s$ metals with the fcc structure, such as $\mathrm{Ag}$ and $\mathrm{Cu}$. We believe that future calculations will enable the examination of general trends in different groups of materials and thus open a way to discover, induce, and control different kinds of nonthermal solid-to-solid phase transitions.

To summarize, we have studied the nonthermal solid-tosolid phase transitions in photoexcited tungsten by extending computational approaches usually used to study pressureinduced phase transitions. We have assumed that electronic excitation can be represented by an electronic temperature, and have studied $T_{e}$-induced dynamical instabilities through $a b$ initio calculations of phonon dispersions and nonthermal forces along structural deformation paths. Our results demonstrate that in tungsten electronic excitation will give rise to nonthermal forces that can drive the initial bcc structure towards the more closed-packed fcc or hcp structures and both of these become dynamically stable. On the basis of the 2TM model and the knowledge of the electron-phonon coupling constant, we estimate that such transient nonthermal fcc/hcp phase could exist for several picoseconds and should be therefore observable using advanced $\mathrm{x}$-ray or electron diffraction methods $[4,5,31]$.

\section{ACKNOWLEDGMENTS}

This work was supported by specially promoted research (24000006) from a Grant-in-Aid for Scientific Research from the JSPS, Japan. We acknowledge the use of the facilities of HECToR/ARCHER, funded by the UK's HPC Materials Chemistry Consortium via EPSRC (EP/F067496), and the UCL Legion High Performance Computing Facility. The authors are grateful to Matthew Watkins for useful discussions and help in the calculations.
[1] J. V. Vechten, R. Tsu, and F. Saris, Phys. Lett. A 74, 422 (1979).

[2] K. H. Bennemann, Ann. Phys. 18, 480 (2009).

[3] K. H. Bennemann, J. Phys.: Condens. Matter 23, 073202 (2011).

[4] G. Sciaini and R. J. D. Miller, Rep. Prog. Phys. 74, 096101 (2011).

[5] R. J. D. Miller, R. Ernstorfer, M. Harb, M. Gao, C. T. Hebeisen, H. Jean-Ruel, C. Lu, G. Moriena, and G. Sciaini, Acta Crystallogr., Sect. A: Found. Crystallogr. 66, 137 (2010).

[6] E. Collet, M. Buron-Le Cointe, and H. Cailleau, J. Phys. Soc. Jpn. 75, 011002 (2006).

[7] E. Collet, M.-H. Lemé-Cailleau, M. Buron-Le Cointe, H. Cailleau, M. Wulff, T. Luty, S.-Y. Koshihara, M. Meyer, L. Toupet, P. Rabiller et al., Science 300, 612 (2003).

[8] M. Lorenc, J. Hébert, N. Moisan, E. Trzop, M. Serl, M. Buron-Le Cointe, H. Cailleau, M. L. Boillot, E. Pontecorvo, M. Wulff et al., Phys. Rev. Lett. 103, 028301 (2009).

[9] K. Nasu, Rep. Prog. Phys. 67, 1607 (2004).

[10] A. Rousse, C. Rischel, S. Fourmaux, I. Uschmann, S. Sebban, G. Grillon, P. Balcou, E. Forster, J. Geindre, P. Audebert et al., Nature (London) 410, 65 (2001).

[11] K. Sokolowski-Tinten, C. Blome, C. Dietrich, A. Tarasevitch, M. Horn von Hoegen, D. von der Linde, A. Cavalleri, J. Squier, and M. Kammler, Phys. Rev. Lett. 87, 225701 (2001).

[12] B. J. Siwick, J. R. Dwyer, R. E. Jordan, and R. Miller, Chem. Phys. 299, 285 (2004).

[13] S. K. Sundaram and E. Mazur, Nat. Mater. 1, 217 (2002).

[14] H. J. Zeiger, J. Vidal, T. K. Cheng, E. P. Ippen, G. Dresselhaus, and M. S. Dresselhaus, Phys. Rev. B 45, 768 (1992).

[15] A. M. Lindenberg, I. Kang, S. L. Johnson, T. Missalla, P. A. Heimann, Z. Chang, J. Larsson, P. H. Bucksbaum, H. C. Kapteyn, H. A. Padmore et al., Phys. Rev. Lett. 84, 111 (2000).

[16] M. Hase, M. Kitajima, S.-i. Nakashima, and K. Mizoguchi, Phys. Rev. Lett. 88, 067401 (2002).

[17] B. Arnaud and Y. Giret, Phys. Rev. Lett. 110, 016405 (2013).

[18] A. Cavalleri, C. Tóth, C. W. Siders, J. A. Squier, F. Ráksi, P. Forget, and J. C. Kieffer, Phys. Rev. Lett. 87, 237401 (2001).

[19] K. Miyano, T. Tanaka, Y. Tomioka, and Y. Tokura, Phys. Rev. Lett. 78, 4257 (1997).
[20] S. Lysenko, A. Rúa, V. Vikhnin, F. Fernández, and H. Liu, Phys. Rev. B 76, 035104 (2007).

[21] T. Kawamoto, Y. Asai, and S. Abe, Phys. Rev. Lett. 86, 348 (2001).

[22] M. Nishino, K. Yamaguchi, and S. Miyashita, Phys. Rev. B 58, 9303 (1998).

[23] E. Collet, H. Watanabe, N. Bréfuel, L. Palatinus, L. Roudaut, L. Toupet, K. Tanaka, J.-P. Tuchagues, P. Fertey, S. Ravy et al., Phys. Rev. Lett. 109, 257206 (2012).

[24] K. Tanimura and I. Akimoto, J. Lumin. 94-95, 483 (2001).

[25] P. Beaud, S. L. Johnson, E. Vorobeva, U. Staub, R. A. De Souza, C. J. Milne, Q. X. Jia, and G. Ingold, Phys. Rev. Lett. 103, 155702 (2009).

[26] J. Kanasaki, E. Inami, K. Tanimura, H. Ohnishi, and K. Nasu, Phys. Rev. Lett. 102, 087402 (2009).

[27] S. Wall, L. Foglia, D. Wegkamp, K. Appavoo, J. Nag, R. F. Haglund, J. Stähler, and M. Wolf, Phys. Rev. B 87, 115126 (2013).

[28] H. Ichikawa, S. Nozawa, T. Sato, A. Tomita, K. Ichiyanagi, M. Chollet, L. Guerin, N. Dean, A. Cavalleri, S.-i. Adachi et al., Nat. Mater. 10, 101 (2011).

[29] K. Tanimura, Phys. Rev. B 70, 144112 (2004).

[30] J. Hohlfeld, S.-S. Wellershoff, J. Gdde, U. Conrad, V. Jhnke, and E. Matthias, Chem. Phys. 251, 237 (2000).

[31] Y. Giret, N. Naruse, S. L. Daraszewicz, Y. Murooka, J. Yang, D. M. Duffy, A. L. Shluger, and K. Tanimura, Appl. Phys. Lett. 103, 253107 (2013).

[32] Z. Lin, L. V. Zhigilei, and V. Celli, Phys. Rev. B 77, 075133 (2008).

[33] K. T. Chan, B. D. Malone, and M. L. Cohen, Phys. Rev. B 86, 094515 (2012).

[34] S. I. Anisimov, B. L. Kapeliovich, and T. L. Perel'man, Zh. Eksp. Teor. Fiz. 66, 776 (1974) [Sov. Phys. JETP 39, 375 (1974)].

[35] D. S. Ivanov and L. V. Zhigilei, Phys. Rev. B 68, 064114 (2003).

[36] S. L. Daraszewicz, Y. Giret, N. Naruse, Y. Murooka, J. Yang, D. M. Duffy, A. L. Shluger, and K. Tanimura, Phys. Rev. B 88, 184101 (2013). 
[37] X. Gonze, B. Amadon, P.-M. Anglade, J.-M. Beuken, F. Bottin, P. Boulanger, F. Bruneval, D. Caliste, R. Caracas, M. Côté et al., Comput. Phys. Commun. 180, 2582 (2009).

[38] X. Gonze, G.-M. Rignanese, M. Verstraete, J.-M. Beuken, Y. Pouillon, R. Caracas, F. Jollet, M. Torrent, G. Zerah, M. Mikami et al., Zeit. Kristallogr. 220, 558 (2005).

[39] D. M. Ceperley and B. J. Alder, Phys. Rev. Lett. 45, 566 (1980).

[40] N. Troullier and J.-L. Martins, Solid State Commun. 74, 613 (1990).

[41] P. Hohenberg and W. Kohn, Phys. Rev. 136, B864 (1964).

[42] W. Kohn and L. J. Sham, Phys. Rev. 140, A1133 (1965).

[43] N. D. Mermin, Phys. Rev. 137, A1441 (1965).

[44] B. G. Walker, C. Molteni, and N. Marzari, J. Phys.: Condens. Matter 16, S2575 (2004).

[45] N. Marzari, D. Vanderbilt, and M. C. Payne, Phys. Rev. Lett. 79, 1337 (1997).

[46] R. M. Wentzcovitch, J. L. Martins, and P. B. Allen, Phys. Rev. B 45, 11372 (1992).

[47] M. Weinert and J. W. Davenport, Phys. Rev. B 45, 13709 (1992).

[48] F. Wagner, T. Laloyaux, and M. Scheffler, Phys. Rev. B 57, 2102 (1998).

[49] G. Grimvall, B. Magyari-Köpe, V. Ozoliņš, and K. A. Persson, Rev. Mod. Phys. 84, 945 (2012).
[50] S. Mazevet, J. Clérouin, V. Recoules, P. M. Anglade, and G. Zerah, Phys. Rev. Lett. 95, 085002 (2005).

[51] E. S. Zijlstra, L. L. Tatarinova, and M. E. Garcia, Phys. Rev. B 74, 220301 (2006).

[52] Y. Giret, A. Gellé, and B. Arnaud, Phys. Rev. Lett. 106, 155503 (2011).

[53] V. Ozolins, Phys. Rev. Lett. 102, 065702 (2009).

[54] K. Einarsdotter, B. Sadigh, G. Grimvall, and V. Ozoliņš, Phys. Rev. Lett. 79, 2073 (1997).

[55] A. Larose and B. N. Brockhouse, Can. J. Phys. 54, 1819 (1976).

[56] V. P. Dmitriev, A. Y. Kuznetsov, D. Machon, H.-P. Weber, and P. Toldano, Europhys. Lett. 61, 783 (2003).

[57] Y. Chen, K. M. Ho, and B. N. Harmon, Phys. Rev. B 37, 283 (1988).

[58] V. Paidar, L. G. Wang, M. Sob, and V. Vitek, Modell. Simul. Mater. Sci. Eng. 7, 369 (1999).

[59] M. Friák and M. Šob, Phys. Rev. B 77, 174117 (2008).

[60] E. S. Zijlstra, A. Kalitsov, T. Zier, and M. E. Garcia, Phys. Rev. X 3, 011005 (2013).

[61] S. L. Daraszewicz, Y. Giret, N. Naruse, Y. Murooka, J. Yang, D. M. Duffy, A. L. Shluger, and K. Tanimura, Appl. Phys. Lett. 105, 023112 (2014).

[62] Y. Yao and D. D. Klug, Solid State Commun. 151, 1873 (2011). 